\title{
A prospective study on the impact of waiting times for radiotherapy for cervical cancer at Charlotte Maxeke Johannesburg Academic Hospital, South Africa
}

\author{
K N Lohlun, MB BCh, MMed, FC Rad Onc (SA); J A Kotzen, BSc, MB BCh, MMed Rad (T); R Lakier, BSc, MMed Rad (T) \\ Division of Radiation Oncology, Charlotte Maxeke Johannesburg Academic Hospital and University of the Witwatersrand, \\ Johannesburg, South Africa
}

Corresponding author: K N Lohlun (kim@kimlohlun.co.za)

Background. Radiotherapy plays a vital role in the management of cervical cancer. However, because of high patient load and limited resources, waiting lists are unacceptably long. This is a highly curable malignancy that often occurs in economically active, relatively young women. The impact of treatment delays on society is therefore disproportionately large when compared with many other malignancies. Delays also impact negatively on the healthcare system and place further stress on an already burdened department.

Objective. To evaluate the potential impact of radiotherapy delays.

Methods. Eighty-one patients requiring radical radiotherapy for cervical cancer were selected. Patients were re-evaluated every 4 weeks while waiting, and again at simulation.

Results. Median delay from first consultation to simulation was 55 days. Longer delays were not statistically correlated to tumour progression. Most of the upstaging occurred around 40 - 65 days. One in four patients received blood transfusions and required hospital admission. Four patients needed haemostatic brachytherapy for bleeding.

Conclusion. A relationship between time waited and disease progression could not be proven. However, numbers were small and statistical tests were probably underpowered. The study does, however, highlight unacceptably long delays for radiotherapy, and a wait of less than 40 days is recommended.

S Afr J Obstet Gynaecol 2015;21(1):6-9. DOI:10.7196/SAJOG.985

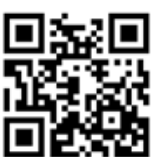

Despite significant advances in screening and management over the past few decades, cervical cancer remains a significant burden, particularly in developing countries, where more than $80 \%$ of cases are diagnosed. ${ }^{[1]}$ Cervical cancer comprises $22.2 \%$ of all cancers in women of sub-Saharan Africa and carries the highest rate of cancer-related mortality. ${ }^{[2]}$

The National Cancer Registry of 2003 showed that cervical cancer is the third leading malignancy in South African (SA) women, after breast and basal cell carcinomas. In black women, it is the leading cancer. ${ }^{[3]}$ The lifetime risk of developing cervical cancer for SA women is one in $31 .{ }^{[4]}$ The burden of disease in developing countries, including SA, therefore remains high. Meanwhile, radiation and other treatment facilities are poorly staffed and poorly equipped, ultimately leading to inability to cope with the large numbers of patients.

Forty per cent of the country's malignancies are diagnosed in Gauteng Province, probably because this region has the highest number of cancer-diagnosing laboratories. At our Gauteng-based institution, we see approximately 750 cases of cervical cancer annually - about $20 \%$ of the total number of cases referred, which is comparable with the $17-20 \%$ noted for national statistics. ${ }^{[4,5]}$

Patients seen at our clinic tend to present with more advanced disease than those in First-World countries. This may be due to several factors, including poor education and lack of awareness, inadequate screening programmes, poor access to healthcare, lack of funding and staff shortages. As radiotherapy is the mainstay of treatment for advanced disease, it plays a vital role in the management of cervical cancer in our country.
Because of large numbers of patients and insufficient machine time to cater for all these patients, Charlotte Maxeke Johannesburg Academic Hospital was, at the time of the study, experiencing waiting lists of up to 16 weeks for patients with cervical cancer. Delays in treatment become a burden not only to the patient and her family, but also to doctors, nurses and other staff. ${ }^{[6]}$ During the delay, patients may require blood transfusions, hospital admissions and/or brachytherapy to stop vaginal bleeding. This increases the financial costs of patient care. On follow-up reviews, it is sometimes noted that the tumour has progressed or that performance status has deteriorated, prompting alterations in radiotherapy from radical to palliative regimens. These unwanted events could possibly be prevented or reduced if waiting times for radiation were decreased. In order to do this, the extent of the problem needs to be properly assessed.

This study was conducted to assess the extent of waiting times for radiation treatment and the potential impact that these delays might have (such as disease progression), as well as to define the need for extra supportive measures.

\section{Methods}

We prospectively looked at waiting times for radiation treatment for cervical cancer and the impact that delay had on the patients and their overall management.

Eighty-one patients, who presented to the department between May 2010 and July 2011, were included in the study. These patients were all scheduled for radical radiotherapy. Patients for palliative radiation regimens, with poor expected overall survival, were excluded, as well as patients who required postoperative radiation. Patients were recruited 
according to the availability of the researcher to recruit in a busy clinical context. This therefore represents a convenience sample with no obvious bias in those recruited that might affect the outcome. Patient characteristics are presented in Table 1.

Written informed consent for both radiation and participation in the study was obtained at first visit. Ethical clearance was obtained from the Human Research Ethics Committee of the University of the Witwatersrand (clearance certificate M10440).

\begin{tabular}{ll}
\multicolumn{2}{l}{ Table 1. Patient characteristics $(\boldsymbol{N}=\mathbf{8 1})$} \\
\hline Characteristic & $\boldsymbol{n}(\%)$ \\
\hline Age group (years) & \\
$20-30$ & $4(5)$ \\
$31-40$ & $11(14)$ \\
$41-50$ & $28(35)$ \\
$51-60$ & $26(32)$ \\
$61-70$ & $10(12)$ \\
$71-80$ & $2(3)$
\end{tabular}

Histological type

Squamous cell carcinoma $\quad 71(88)$

Adenocarcinoma $6(7)$

Adenosquamous carcinoma $1(1)$

Basaloid squamous carcinoma $\quad 1(1)$

Unknown

Tumour grade

I (well differentiated)

II (moderately differentiated)

III (poorly differentiated)

IV (undifferentiated)

Unknown

Tumour stage

$\begin{array}{ll}\mathrm{IB}_{1} & 1(1) \\ \mathrm{IB}_{2} & 2(3) \\ \mathrm{IIA} & 1(1) \\ \mathrm{IIB} & 44(54) \\ \text { IIIA } & 2(3) \\ \text { IIIB } & 31(38)\end{array}$

HIV status

Negative

Positive

ECOG performance status

0

1

2

Hospital level

Secondary

Tertiary
Patients were reviewed every 4 weeks until simulation. At each visit, the following parameters were checked: any blood transfusions received, number of units received, hospital admissions, vaginal bleeding, and bladder or bowel complaints. The administration of haemostatic brachytherapy (because of excessive bleeding or the requirement of multiple blood transfusions) was also documented. A physical examination was done. Special note was taken of the overall performance status, and of local tumour extent or progression. The same patient review was repeated at the simulator.

\section{Statistical methods}

Statistical tests were performed using IBM SPSS version 20.0. A $p$-value of $<0.05$ was considered statistically significant

A one-sample Kolmogorov-Smirnov test was used to assess whether time from consultation to simulation was normally distributed. As results indicated non-normality $(p<0.001)$, median values were used instead of mean values, which would not have provided a relevant reflection of the average patient's experience because of higher than normally expected values.

The Mann-Whitney $U$-test was used to determine statistical significance for differences in frequency distributions of waiting times, with exact significance testing used if numbers were low $(n<30)$.

Kaplan-Meier estimates were used to determine length of time elapsed while waiting for simulation before stage progression occurred. These methods, using logranks, were also employed to evaluate differences in stage progression according to original tumour stage, HIV status and histological grade.

\section{Results}

Median time from first consultation to simulation was just short of 2 months (55 days), as demonstrated in Table 2. The wait from simulation to commencement of treatment took on average another 19 days. Median total delay in starting treatment as a consequence of departmental waiting lists was therefore 74 days. However, this does not take into account delays due to other factors, such as patients defaulting or being unreachable by both telephone and telegram.

The maximum delay from day of first consultation to simulation was 211 days. This was due to patient factors rather than departmental delay. If we exclude the top five outliers of the valid population $(6 \%)$, who waited longer than 70 days from first consultation to simulation, the median waiting time would still be 55 days.

One in four patients received blood transfusions while waiting for simulation. However, the median haemoglobin level was only $11.1 \mathrm{~g} / \mathrm{dL}$, with the minimum referral haemoglobin being as low as $3.8 \mathrm{~g} / \mathrm{dL}$. The Mann-Whitney $U$-test showed no statistical difference in waiting time between those who received blood transfusions and those who did not $(p=0.70)$.

Of the 79 patients who returned for follow-up, 21 (27\%) were admitted to hospital during the wait. Most of the admissions were for anaemia, where a blood transfusion was required. Only three were admitted and did not receive a blood transfusion. Two of these were admitted for transport reasons, as they had no other means to get to the radiation unit. The third patient said she was admitted for 'an operation to treat the cancer', but this was not done. As she had already presented with stage IIB cancer, this admission was most likely the result of a mix-up or miscommunication problem. Longer waiting times from first consultation to simulation did not predict for hospital admission $(p=0.84)$.

Four $(5 \%)$ of the participants were given brachytherapy for haemostasis while awaiting treatment.

Table 2. Delay times

\begin{tabular}{llllll}
\hline & \multicolumn{5}{c}{ Time interval (days) } \\
\cline { 2 - 6 } & Mean & Median & Min. & Max. & SD \\
\hline Diagnosis to consultation $(n=81)$ & 45 & 36 & 6 & 274 & 34 \\
Consultation to simulation $(n=77)$ & 54 & 55 & 18 & 211 & 25 \\
Simulation to treatment $(n=77)$ & 20 & 19 & 1 & 76 & 11 \\
Diagnosis to treatment $(n=77)$ & 119 & 108 & 65 & 324 & 39 \\
Consultation to treatment $(n=77)$ & 73 & 74 & 30 & 222 & 26 \\
SD $=$ standard deviation. & & & & &
\end{tabular}


From the time of first consultation to simulation, 35 (44\%) of the 79 evaluable patients had progression of their tumours. Twenty-two (28\%) were upstaged. Nineteen (24\%) required modification of their planned treatments to a less radical regimen (e.g. hypofractionated radiation), as per departmental protocol. It was suspected that one patient had developed a vesicovaginal fistula at simulation review, which would have upstaged the tumour to stage IVA. However, this was not verified and the patient's good performance status suggested a fair prognosis. She was therefore given radical radiation as planned.

In terms of both tumour progression and stage changes, the time waited from first consultation to simulation was not statistically significant, with $p$-values of 0.08 and 0.21 , respectively. However, median values were slightly higher for the average patient whose disease became more advanced, with median waiting times of 55 and 53 days for tumours that had progressed v. those that had not, and 57 and 55 days for tumours that were upstaged $v$. those that were unchanged.

Fig. 1 shows a Kaplan-Meier plot of time from consultation to simulation against the proportion of patients whose tumour stage did not change. The steep drop in the curve indicates that the majority of those patients whose tumours progressed and were upstaged, experienced these changes between 40 and 65 days of waiting.
Neither HIV status nor histological differentiation (grade) was significant with regard to stage progression, with log-rank test $p$-values of 0.20 and 0.18 , respectively.

Only five patients (6\%) were evaluated as having a deterioration in Eastern Cooperative Oncology Group (ECOG) performance status, with the majority staying at an ECOG of I. There were too few changes in performance status to evaluate significance in respect of time waited.

Ten of the 81 patients in the study either missed (defaulted) one or more of their monthly reviews while waiting for a simulation date, or declined treatment at some point. Reasons for defaulting were often unclear, with some stating social problems and others giving no real explanation. Again, numbers were too low to do statistical tests on those who had defaulted. The patients who defaulted at some time had median waiting times from first consultation to simulation of 67 days, whereas those who did not default waited a median time of 55 days.

Of the patients who were treated or could be reached, none died while awaiting radiation. However, at least one patient progressed to stage IV disease and was given palliative radiation.

\section{Discussion}

The median delay from first consultation until commencement of treatment was 74 days. At the time of writing a protocol

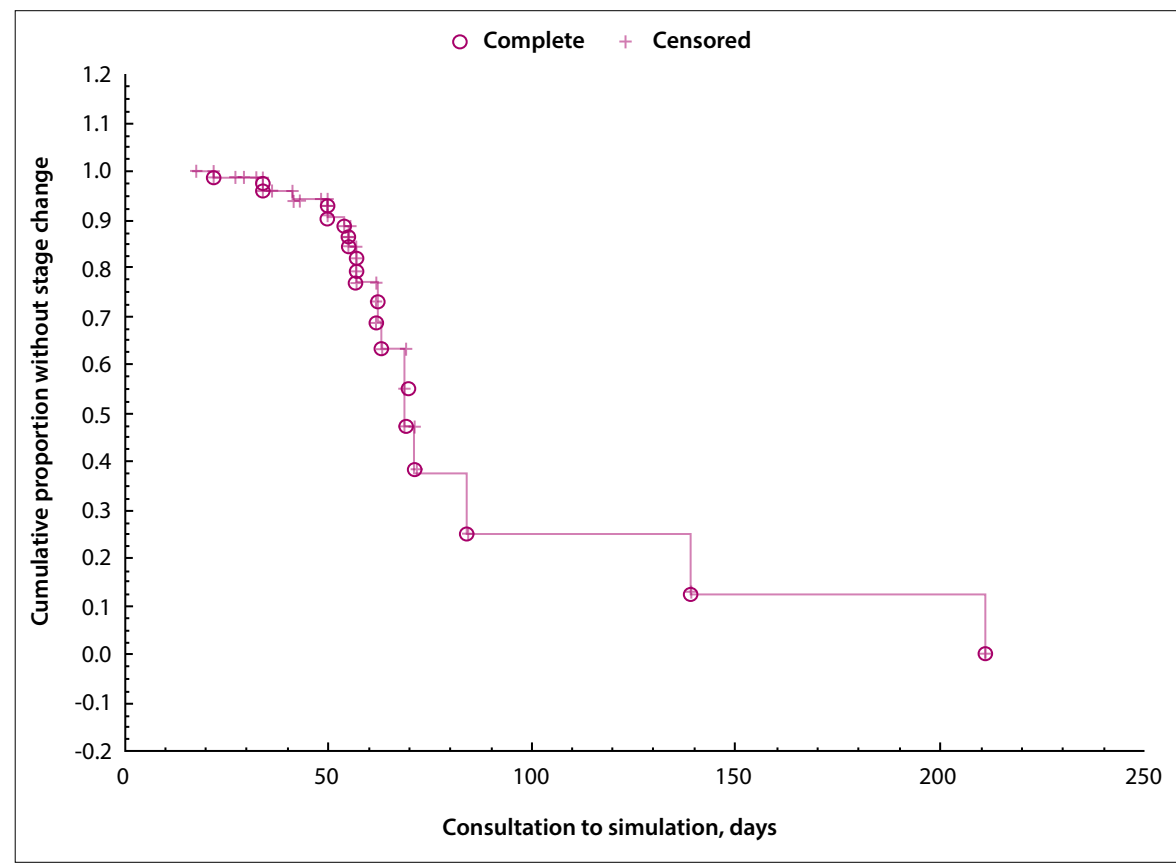

Fig. 1. Kaplan-Meier plot of time from consultation to simulation against the proportion of patients whose tumour stage did not change. for this study, it was noted that some patients had been waiting up to 4 months for simulation alone. The reason for this discrepancy was the introduction of a hypofractionated radiation protocol, which was introduced with the aim of reducing waiting times by using higher, but fewer, daily doses of radiation, and thereby decreasing the overall duration of treatment.

Mackillop et al..$^{[7]}$ found that the median waiting time for patients with cervical cancer in Ontario was 27.2 days from diagnosis to commencement of radiotherapy. They observed, as with this study, that most of the wait lay in the period following the first consultation, but gave no figure for this time interval. We therefore compared their median total time of 27.2 days with our median total time of 108 days from diagnosis to treatment. Although Mackillop et al. cited waiting times as a problem in Ontario, it is clear that delays in the Canadian setting are in no way comparable with those at our institution, where the wait is four times as long.

At the time of simulation, $27 \%$ of the study patients had tumours that had progressed to a more advanced stage since the time of first consultation. This would, theoretically, translate to a poorer prognosis and decreased survival, given that 5 -year survival rates are roughly $80 \%, 65 \%$ and $40 \%$ for stages I, II and III, respectively. ${ }^{[8]}$ A longer follow-up of these patients would be required to assess lives lost as a result of treatment delay. The study, however, failed to show that tumour progression was increased with a longer delay.

Mackillop et al. ${ }^{[9]}$ concluded that there is no theoretical reason to believe that any delay is safe and the best course would be to adopt an ASARA principle, i.e. to keep delays 'As Short As Reasonably Achievable', this being modelled from the ALARA principle of keeping radiation doses 'As Low As Reasonably Achievable. While this principle is both logical and sensible, it may be of benefit to define specific goals towards which radiation departments can aim.

In this study, progression of the tumours that were upstaged was noted between 40 and 65 days, using Kaplan-Meier plots. Fortin et al. ${ }^{[10]}$ looked at time waited from consultation to start of treatment for radically treated head-and-neck tumours, which are considered to have similar doubling times to cervical cancers. Their study demonstrated diminished locoregional control and overall survival for 
patients who waited longer than 40 days for radiation treatment. These patients had a $15 \%$ lower survival rate at 3 years. Although we looked at time from first consultation to simulation, rather than time to start of treatment, the trend of the plot (Fig. 1) implies that should patients have been re-evaluated at the start of treatment, the sharp drop between 40 and 65 days would still be relevant. This study, in accordance with Fortin et al.'s study, therefore supports the conclusion that a delay of more than 40 days may compromise tumour control and subsequent patient survival.

Bleeding per vagina is a common clinical presentation of cervical cancer. The longer patients wait for radiation treatment, the higher the chances that they will require multiple blood transfusions or haemostatic brachytherapy to stop the bleeding. In this study, one in four patients received a blood transfusion while waiting for simulation. The cost of one unit of red cell concentrate is ZAR1 369.39 in the state sector, while blood administration sets cost a further ZAR796.05 each. ${ }^{[1]}$ This is over and above the price of the hospital stay.

Most of the patients who were transfused were admitted to hospital. According to statistics compiled by economist Schüssler, only $2 \%$ of the cost of a public sector admission is charged to the patient. The remaining $98 \%$ is borne by the taxpayer. ${ }^{[12]}$ Zietsman $^{[12]}$ concludes, in his analysis of hospital costs, that while public healthcare appears cheaper than private care, the opposite is actually true when one takes into consideration the overall cost to the economy and the effectiveness of providing that care.

Brachytherapy for haemostasis is limited to only a few hospitals, and these facilities are already burdened with high patient volumes and insufficient treatment time. Four patients received haemostatic brachytherapy for bleeding.

\section{Conclusion}

Carcinoma of the uterine cervix remains a problem in the developing world, particularly in sub-Saharan Africa. Despite the large burden of disease, treatment facilities are still lacking. Supply is unable to keep up with demand, and waiting lists for radiotherapy are unacceptably long. This has consequences not only for patients' health and life expectancy, but also on the healthcare system and society in general, given that these patients are often economically active individuals.
This study was conducted to assess the potential impact that treatment delays may be having on the healthcare system, the patients and their disease. Results did not reach statistical significance. However, median delay times of 10 weeks from first consultation to treatment, $43 \%$ of patients having progressive disease and $27 \%$ having tumours upstaged are all figures of concern. Although tumour progression could not be correlated to time waited, it was noted that most of the tumours that were upstaged were upstaged between 40 and 65 days. Reasonable goals for the department would therefore be to decrease the wait from first consultation to simulation to less than 40 days.

This study provides a basic overview of times waited for radiotherapy for cervical cancer at Charlotte Maxeke Hospital. This will provide a platform for future comparisons and for assessment of progress and improvement within the department. Further studies may look at quality of life for patients awaiting treatment, patient satisfaction with the care received, staff and equipment requirements for improving waiting times, and outcomes for patients who were treated using the shortened hypofractionated protocol.

1. Denny L Cytological screening for cervical cancer prevention. Best Pract Res Clin Obstet Gynaecol 2012;26(2):189-196. [http://dx.doi.org/10.1016/j.bpobgyn.2011.08.001]

2. Anorlu RI. Cervical cancer: The Sub-Saharan African perspective. Reprod Health Matters 2008;16(32):41-49. [http://dx.doi.org/10.1016/S0968-8080(08)32415-X]

3. National Cancer Registry. Incidence of histologically diagnosed cancer in South Africa. National Institute for Occupational Health (database online). 2003. http://www.nioh.ac.za/assets/files/ Cancer\%20tables\%202003.pdf (accessed 5 March 2014).

4. Mqoqi N, Kellet P, Sitas F, et al. Incidence of histologically diagnosed cancer in South Africa, 1998-1999. National Cancer Registry of South Africa. Johannesburg: National Health Laboratory Service, 2004:viii, 23 .

5. Denny L. Cervical cancer: The South African perspective. Int J Gynaecol Obstet 2006;95(Supp 1):S211-S214. [http://dx.doi.org/10.1016/S0020-7292(06)60036-2]

6. Dische S. Tumour growth while waiting: Does it really matter? Clin Oncol (R Coll Radiol) 2000;12:139. [http://dx.doi.org/10.1007/s001740070054]

7. Mackillop WJ, Fu H, Quirt CF, et al. Waiting for radiotherapy in Ontario. Int J Radiat Oncol Biol Phys 1994;30(1):221-228. [http://dx.doi.org/10.1016/0360-3016(94)90538-X]

8. Cannistra SA, Niloff JM. Cancer of the uterine cervix. N Engl J Med 1996;334:1030-1038. [http:// dx.doi.org/10.1056/NEJM199604183341606]

9. Mackillop WJ, Bates JH, O'Sullivan B, et al. The effect of delay in treatment on local control by radiotherapy. Int J Radiat Oncol Biol Phys 1996;34(1):243-250. [http://dx.doi.org/10.1016/03603016(95)02049-7]

10. Fortin A, Bairati I, Albert M, et al. Effect of treatment delay on outcome of patients with earlystage head-and-neck carcinoma receiving radical radiotherapy. Int J Radiat Oncol Biol Phys 2002;52(4):929-936. [http://dx.doi.org/10.1016/S0360-3016(01)02606-2]

11. South African National Blood Service. SANBS State Patients Price List 2012. http://www.sanbs.org. za/images/pdfdocs/state\%20patients\%202012.pdf (accessed 25 January 2013).

12. Zietsman G. Why Motsoaledi's wrong about private sector hospital costs. Politicsweb. 13 April 2012. http://www.politicsweb.co.za/politicsweb/view/politicsweb/en/page71619?oid= 292572\&sn=Detail\&pid=71619 (accessed 5 March 2014) 